\title{
Measuring the Dzyaloshinskii-Moriya interaction in a weak ferromagnet
}

\author{
V. E. Dmitrienko', E. N. Ovchinnikova ${ }^{2}$, S. P. Collins ${ }^{3 \star}{ }^{1}$, G. Nisbet ${ }^{3}$, G. Beutier ${ }^{4}$, Y. O. Kvashnin ${ }^{5}$, \\ V. V. Mazurenko ${ }^{6}$, A. I. Lichtenstein ${ }^{7}$ and M. I. Katsnelson ${ }^{6,8}$
}

\begin{abstract}
Magnetism-the spontaneous alignment of atomic moments in a material-is driven by quantum mechanical exchange interactions that operate over interatomic distances. Some magnetic interactions cause ${ }^{1,2}$, or are caused by ${ }^{3,4}$, a twisting of arrangements of atoms. This can lead to the magnetoelectric effect, predicted to play a prominent role in future technology, and to the phenomenon of weak ferromagnetism, governed by the so-called Dzyaloshinskii-Moriya interaction ${ }^{5-8}$. Here we determine the sign of the latter interaction in iron borate $\left(\mathrm{FeBO}_{3}\right)$ by using synchrotron radiation. We present a novel experimental technique based on the interference between two $X$-ray scattering processes, where one acts as a reference wave. Our experimental results are validated by state-ofthe-art $a b$ initio calculations. Together, our experimental and theoretical approaches are expected to open up new possibilities for exploring, modelling and exploiting novel magnetic and magnetoelectric materials.
\end{abstract}

There is considerable mystery behind the origins of complicated structures. Although the dominant short-range interactions that allow the building blocks to grow are well understood, the much more subtle forces that lead to a particular twisting at larger lengthscales, such as chiral biological molecules and liquid crystals ${ }^{9}$, and canted magnetic systems ${ }^{3}$, remain subjects of topical debate. In this Letter we seek to address this question for the case of magnetism. Our main findings are twofold: first, we demonstrate a novel and elegant experimental method for exploring magnetic materials with weak relativistic spin-orbit interactions, and second, we present a state-of-the-art quantum-mechanical many-body approach to the detailed description of such interactions in crystals. As a touchstone example we selected crystalline iron borate $\left(\mathrm{FeBO}_{3}\right)$, which is a strongly correlated electron system with a relatively simple crystal structure, nonetheless allowing a nontrivial canted and locally twisted magnetic ordering pattern. Taken together, these two strands demonstrate that modern condensed matter theory is capable of determining the elusive sign of the Dzyaloshinskii-Moriya interaction, and is thus able to elucidate the mechanism for coupling electric and magnetic degrees of freedom in magnetoelectric multiferroics, and to begin to predict the properties of this important class of materials.

The interactions between atomic magnetic moments (or spins) are not direct, but mediated by the intervening matter. Coupling can be diminished through screening ${ }^{10}$, or enhanced, for example, by superexchange via oxygen atoms ${ }^{11}$. Moreover, the coupling is a property of the material and, according to Neumann's principle, must therefore possess all of its symmetries. The most general form of the bilinear coupling energy between two spins contains a scalar (isotropic) exchange term, exchange anisotropy (which we will neglect for the present discussion) and an antisymmetric term that reverses with permutation of the spin indices. The latter is the Dzyaloshinskii-Moriya interaction, which can be expressed in terms of a Dzyaloshinskii-Moriya vector, $\mathbf{D}$, and the vector product of spins, leading to a spin-dependent energy (for classical spins),

$$
\Delta E=\sum_{m \neq n} J_{m n} \mathbf{S}_{m} \cdot \mathbf{S}_{n}+\sum_{m \neq n} \mathbf{D}_{m n} \cdot\left[\mathbf{S}_{m} \times \mathbf{S}_{n}\right]-\mu_{\mathrm{B}} g S \sum_{m} \mathbf{H} \cdot \mathbf{S}_{m}
$$

where the summations run over all magnetic atoms. $\mathbf{S}_{m}$ is a unit vector along the direction of the $m$ th spin of magnitude $S, \mu_{\mathrm{B}}$ is the Bohr magneton and $g \approx-2$ is the gyromagnetic ratio. Both the exchange coefficients $J_{m n}$ and the Dzyaloshinskii-Moriya vectors $\mathbf{D}_{m n}$ depend on the relative positions of the magnetic atoms. The dominant first term in equation (1) prefers parallel/antiparallel coupling of spins (depending on the sign of $J_{m n}$ ), whereas the second term favours an orthogonal alignment, producing a small twist, or canting, of the atomic moments.

In $3 d$ transition metal oxides it is usually necessary to consider only the nearest neighbours, because these dominate the exchange interactions. The Dzyaloshinskii-Moriya interaction is typically a few per cent of the isotropic term $(\sim 0.1 \mathrm{meV}$, compared to $\sim 10 \mathrm{meV}$ ), producing just a modest canting. Nevertheless, the effect is important. A spontaneous rearrangement of atoms to favour the Dzyaloshinskii-Moriya interaction (often called the inverse Dzyaloshinskii-Moriya effect) can produce a large electric polarization in magnetoelectric materials ${ }^{1,2}$. In so-called weak ferromagnets, canting of the otherwise collinear antiferromagnetic arrangement leads to a small net ferromagnetic polarization that couples strongly to an external magnetic field ${ }^{12}$.

Chiral spin structures in globally centrosymmetric crystals can be determined by using polarized neutron scattering ${ }^{13,14}$ or circularly polarized X-rays ${ }^{15}$. Although such structures are of topical interest owing to the presence of skyrmion lattices, the magnetoelectric effect and so on, the underlying exchange coupling is identical to the classical problem of weak ferromagnetism. Moreover, the latter is a particularly attractive test case for developing new theoretical techniques, as the magnetic structures do not exhibit long (or infinite) magnetic periodicities. Unfortunately, standard

\footnotetext{
${ }^{1}$ A. V. Shubnikov Institute of Crystallography RAS, Moscow 119333, Russia, ${ }^{2}$ Faculty of Physics, M. V. Lomonosov Moscow State University, Leninskie Gory, Moscow 119991, Russia, ${ }^{3}$ Diamond Light Source Ltd, Diamond House, Harwell Science and Innovation Campus, Didcot, Oxfordshire OX11 ODE, UK, ${ }^{4}$ SIMaP, CNRS, Grenoble-INP \& UJF, BP 75, Saint-Martin d'Hères cedex 38402, France, ${ }^{5}$ European Synchrotron Radiation Facility, 6 Rue Jules Horowitz, BP220, Grenoble Cedex 38043, France, ${ }^{6}$ Department of Theoretical Physics and Applied Mathematics, Ural Federal University, Mira str. 19, Ekaterinburg 620002, Russia, ${ }^{7}$ I. Institut für Theoretische Physik, Universität Hamburg, Jungiusstraße 9, Hamburg D-20355, Germany, ${ }^{8}$ Radboud University Nijmegen, Institute for Molecules and Materials, Heyendaalseweg 135, AJ Nijmegen NL-6525, The Netherlands. *e-mail: steve.collins@diamond.ac.uk
} 
a

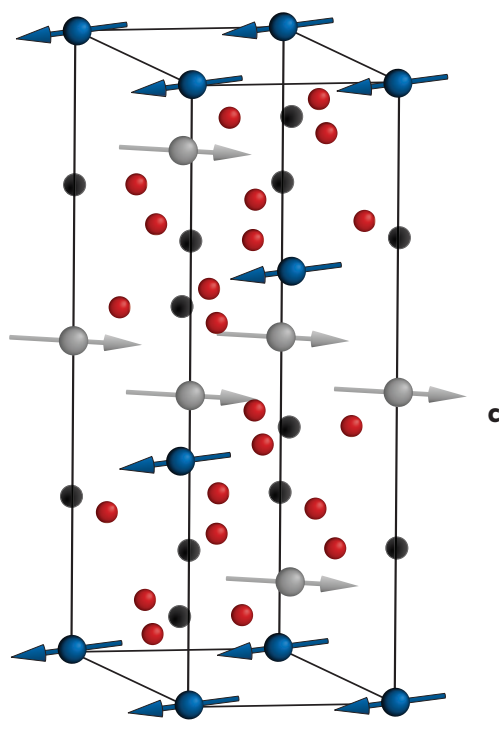

b $\mathrm{Fe}_{\mathrm{A}}$

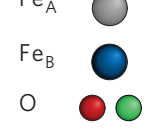

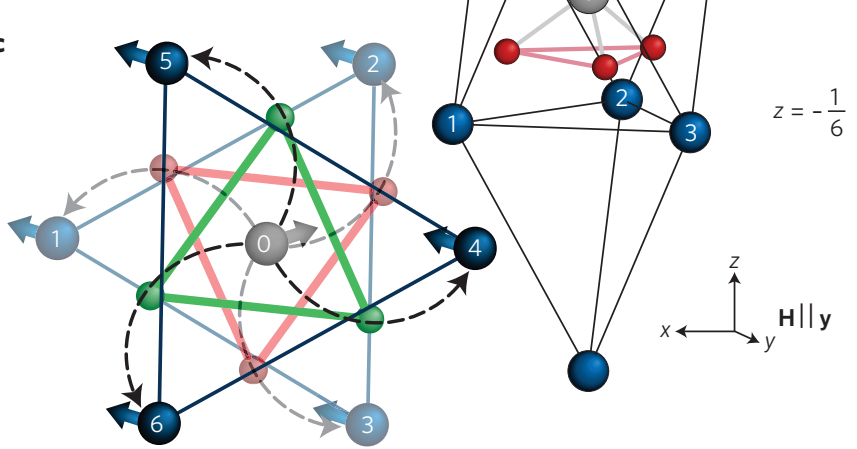

Figure 1 | Atomic and magnetic order in $\mathrm{FeBO}_{3}$. a, A magnetic (hexagonal) unit cell, showing oxygen atoms (red), boron atoms (black), and two symmetry-related magnetic iron sublattices (blue and grey) with moments tilted between the two. $\mathbf{b}$, The local environment of one of the grey (A-site) Fe atoms, showing neighbouring B-site Fe atoms (blue). The upper and lower oxygen triangles are coloured green and red, and boron atoms are removed for clarity. c, The same structure viewed from the top, highlighting the twisted superexchange paths from the A-site Fe atom to the upper Fe layer (dark blue) and the lower layer (pale blue) via the oxygen triangles.

techniques do not reveal the phase of the magnetic structures with respect to the twisting of the atomic arrangement and so the direction of magnetic twist in weak ferromagnets cannot be determined and has remained an unsolved problem.

Important symmetry restrictions on the Dzyaloshinskii-Moriya vector have been discussed since it was initially introduced more than fifty years ago ${ }^{5-8}$. In the case of $\mathrm{FeBO}_{3}$, with crystal symmetry $R \overline{3} c$, there are two iron atoms at the $2 b$ inversion centres, two boron atoms at the $2 a$ positions, and six oxygens at $6 e$ sites. If the oxygen atoms were absent, or positioned exactly between two neighbouring iron atoms, the symmetry would be $R \overline{3} m$, which does not allow for the Dzyaloshinskii-Moriya interaction. It is therefore the small displacement of oxygen atoms, striving towards a close-packed structure, that drives iron borate into the observed complex magnetic ordering pattern shown in Fig. 1. Iron atoms form ferromagnetic layers with their magnetic moments aligned in plane, with the layers stacked in alternating but not exactly opposite directions. (The antiferromagnetic pattern produces weak pure magnetic diffraction at points in reciprocal space that are otherwise forbidden by spacegroup symmetry.) A closer examination of the $\mathrm{FeBO}_{3}$ crystal structure reveals that each $\mathrm{Fe}$ atom is connected to six equivalent nearest $\mathrm{Fe}$ neighbours: three in the plane above and three below. The six DzyaloshinskiiMoriya vectors linking these $\mathrm{Fe}$ atoms are related by symmetry and, when summed, lead to a resultant vector along $z$. It follows from equation (1) that the Dzyaloshinskii-Moriya vector of this type induces a twist between $\mathrm{A}$ and $\mathrm{B}$ spins in the $x y$ plane, but the symmetry alone cannot say whether this twist will be left-handed or right-handed. The absolute sign of the local twist can be found both experimentally and theoretically using the techniques described here.

The present experimental technique relies on the fact that the weak ferromagnetic moment is perpendicular to the opposing antiferromagnetic components (Fig. 1). As the former can be turned

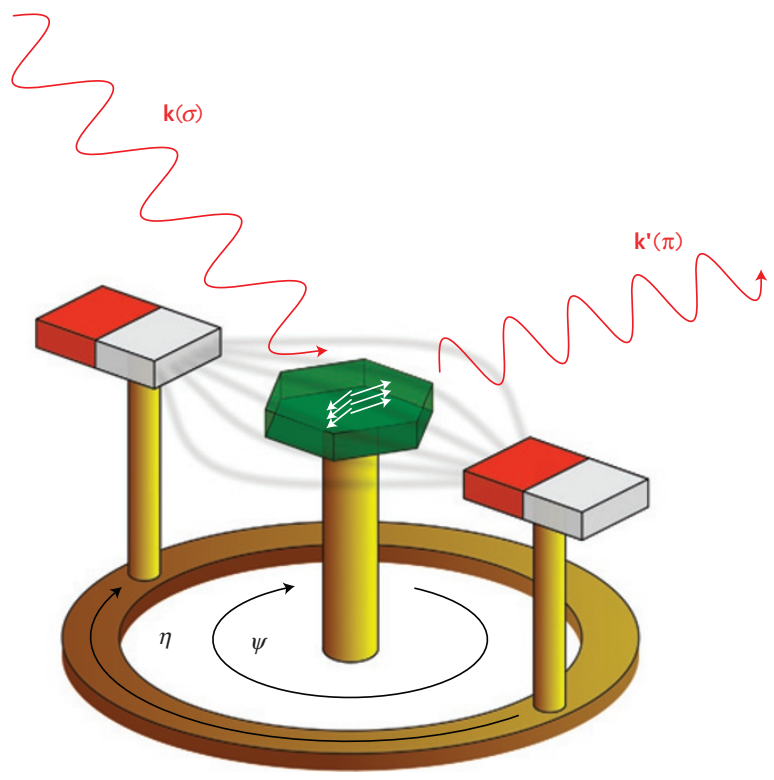

Figure 2 | A schematic view of the experiment carried out on $\mathrm{FeBO}_{3}$. The incident and scattered $X$-ray beams are shown, along with the $\mathrm{FeBO}_{3}$ crystal, indicating weak ferromagnetic moments aligned with the applied magnetic field. The sample and field were rotated about a common axis so that $\psi=0$ and $\eta=0$ correspond to the crystallographic (100) hex axis and the field direction (from south to north pole of the magnet assembly), directed along $\mathbf{k}+\mathbf{k}^{\prime}$, respectively.

with a small external field (Fig. 2), the dominant antiferromagnetic structure is 'dragged' around to follow it, providing an elegant alternative to rotating the entire crystal, thus avoiding experimental artefacts such as multiple X-ray diffraction. 


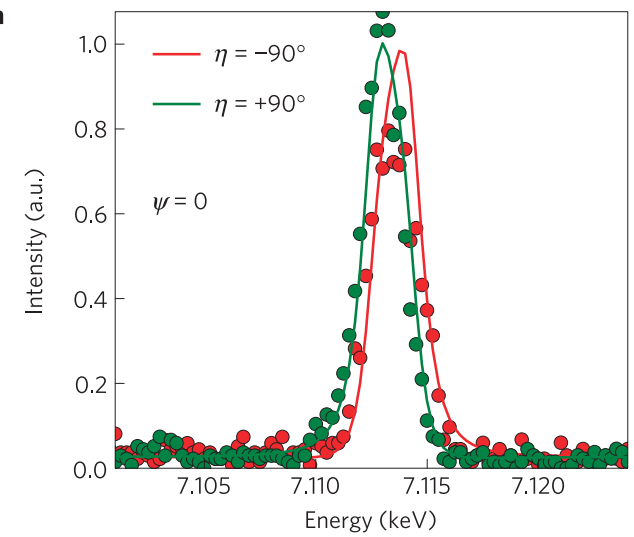

b
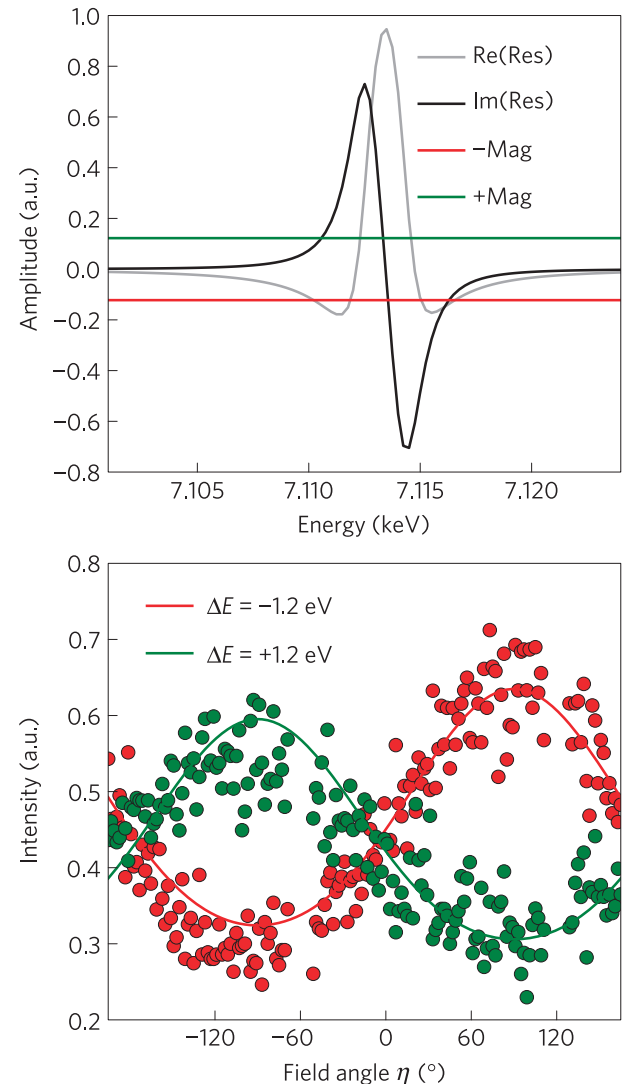
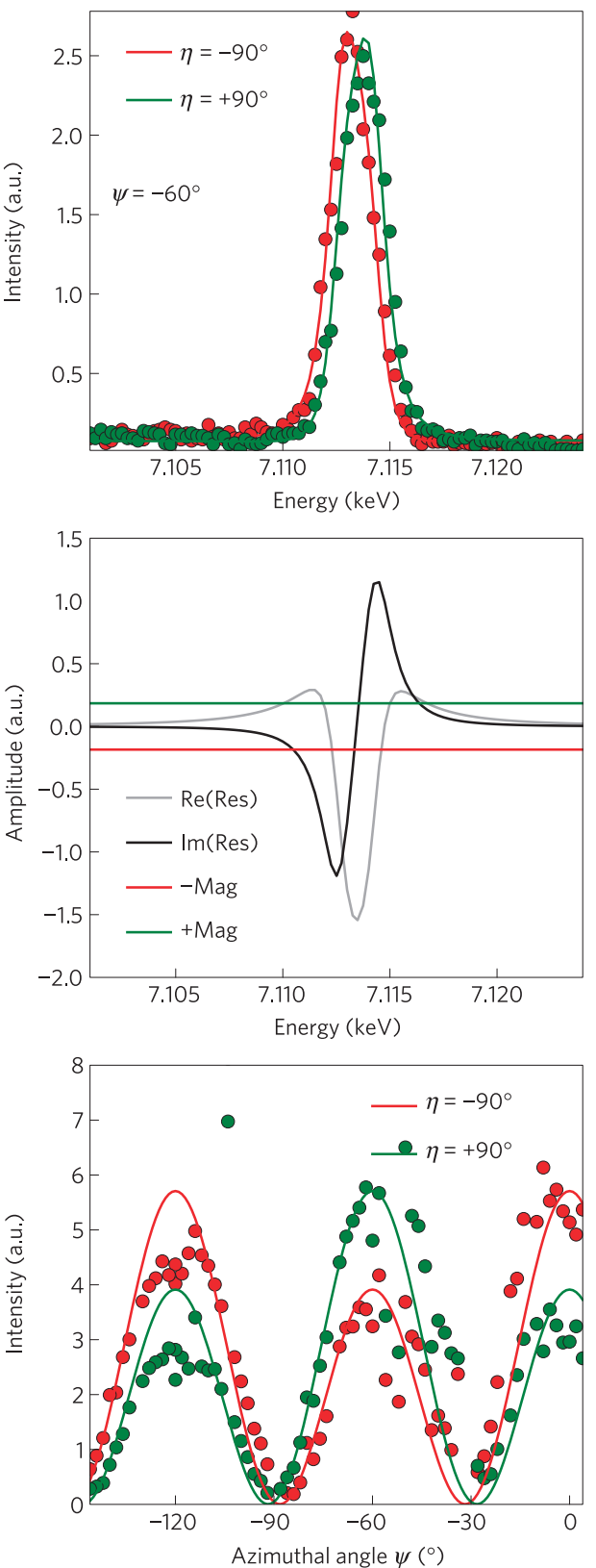

Figure 3 | The major experimental results and simulations for $\mathrm{FeBO}_{3}$. Fits are derived from the coherent sum of the magnetic and resonant amplitudes, with the latter based on a 'double-resonance' model (see Supplementary Information). $\mathbf{a}$, A remarkable shift in the X-ray resonance energy is observed on rotation of the magnet $\left(\eta= \pm 90^{\circ}\right.$ ). The shift reverses as the sample azimuth is rotated from $\psi=0$ to $\psi=-60^{\circ}$ (reversing the phase of the resonant amplitude), as is evident in both experimental data and model calculations. $\mathbf{b}$, The resonant and magnetic amplitudes used to fit the data in $\mathbf{a}$. The origin of the energy jump can be understood by considering interference between the constituent amplitudes. The magnetic amplitude is in phase or antiphase with the imaginary part of the resonance amplitude (black lines), causing constructive: (destructive) interference on the low: (high) energy side of the resonance, depending on the relative phase of the magnetic and resonant scattering amplitudes. c, The same phase of the magnetic scattering gives very good agreement with intensity measurements versus field angle (left), with the resonance amplitude reversed by shifting the energy above and below the resonant centre $(\Delta E= \pm 1.2 \mathrm{eV})$. Right: the intensity variation with azimuthal angle and opposite magnetic amplitudes $\left(\eta= \pm 90^{\circ}\right)$. In $(\mathbf{a})$ and $(\mathbf{c})$, circles represent experimental data and solid lines are fits.

To determine the sign of the Dzyaloshinskii-Moriya interaction, we must determine whether the rotation of the spins is in the same sense as the rotation of the oxygen triangles, or opposite. Unfortunately, the standard techniques for characterizing antiferromagnetic structures-polarized neutron or X-ray diffraction-do not help: the sign of the twist appears in the phase of the diffracted wave, which is lost in an intensity measurement-an aspect of the famous 'phase problem' of crystallography. Borrowing from the ideas behind holography, it was recently suggested by some of $u^{16}$ that the sign of the Dzyaloshinskii-Moriya vector could be measured with resonant X-ray diffraction by observing interference between the resonant ${ }^{17}$ and magnetic ${ }^{18}$ scattering amplitudes. The resonant scattering process adopted is a rather exotic one, involving pure electric quadrupole events (that is, beyond the usual dipole approximation). However, in recent years, such phenomena have been studied in detail and are now extremely well understood ${ }^{19,20}$. Its phase and amplitude vary rapidly with photon energy, being significant only very close to the Fe $\mathrm{K} \mathrm{X}$-ray absorption pre-edge 
energy of $7.11 \mathrm{keV}$, and it has a complex dependence on both photon polarization and the azimuthal rotation of the sample about the normal to the diffracting planes ( $\psi$-angle). Moreover, both the resonant and magnetic scattering signals appear at the same Bragg reflection positions- $(h k l)=(0,0,6 n+3)$-that are 'forbidden' for the vastly stronger charge scattering processes, and have comparable amplitudes to each other, maximizing the effects of interference. The sign and amplitude of the magnetic scattering signal depends on the spin direction, which can be rotated with a magnetic field, whereas those of the resonant scattering depend on the precise X-ray energy and azimuthal orientation of the crystal. We thus expect control of the amplitude and phase of both the magnetic scattering and resonant reference wave, allowing the phase of the magnetic scattering to be determined. Details of the magnetic and resonant scattering amplitudes are given in Supplementary Information.

Three types of measurement are presented. The first shows a remarkable effect: an apparent jump in the energy of the resonant scattering peak as the magnetic motif is rotated by $180^{\circ}$ - the result of constructive (destructive) interference on the low (high) energy side of the resonance (Fig. 3). The opposite jump was observed when the phase of the resonant scattering was reversed by changing the sample $\psi$ angle. Both jumps are reproduced by our ab initio calculations, which make a definite prediction for the sign of the Dzyaloshinskii-Moriya interaction, the phase of the magnetic scattering amplitude, and thus the direction of the jump. The second measurement shows the intensity, measured as a continuous rotation of the field angle, for the low and high energy side of the resonance. For the final measurement, the sample azimuthal angle was varied continuously, with a fixed photon energy and field applied in two opposite directions, transverse to the incident beam. In all cases, the phase of the magnetic scattering whose reversal would convert red to green lines and vice versa were consistent, completely unambiguous, and in agreement with the calculations.

One of the main goals of the present work was to demonstrate that the sign of the Dzyaloshinskii-Moriya interaction can be determined reliably not only by experiment but also theoretically. To this end we have performed first-principles calculations by using the local density approximation incorporating the on-site Coulomb interaction $U$ and the spin-orbit coupling ${ }^{21,22}$ $(\mathrm{LDA}+\mathrm{U}+\mathrm{SO})$. Our calculations predict (see Supplementary Information) that the lowest energy stable magnetic structure is precisely the one observed experimentally and shown in Fig. 1. Furthermore, we predict that the magnetic twist between adjacent layers is in the same direction as the twist of the oxygen triangles. This is the basis of the calculated curves in Fig. 3, and is thus very clearly confirmed by experiment.

Because the vector product $\left[\mathbf{S}_{\mathrm{A}} \times \mathbf{S}_{\mathrm{B}}\right]$ in equation (1) is parallel to the $z$ axis, and the corresponding Dzyaloshinskii-Moriya interaction must reduce the energy of the system, we can deduce that $D_{\mathrm{AB}}^{z}$ is negative. The absolute value of the DzyaloshinskiiMoriya interaction energy is readily estimated from the measured canting angle $\left(0.9^{\circ}\right)$ and isotropic exchange interaction: $\left|D_{\mathrm{AB}}^{z}\right|=2 J\left|S_{y} / S_{x}\right|=0.33 \mathrm{meV}$. Here $J$ is approximately $10.3 \mathrm{meV}$.

The determination of the $J_{m n}$ and $\mathbf{D}_{m n}$ parameters taking into account hybridization, correlation, temperature and spin-orbit coupling effects is a complex methodological and computational problem requiring a whole arsenal of numerical techniques ${ }^{8,23-25}$. Here, we outline a second, and very general next-generation method that has a simple formulation and captures all the important electronic and magnetic excitation effects ${ }^{26}$. The resulting expression for the correlated band Dzyaloshinskii-Moriya interaction can be applied to a wide range of materials and is given by

$$
\mathbf{D}_{m n}=-\frac{i}{2} \operatorname{Tr}_{L, \sigma}\left\{N_{n m}\left[\hat{\mathbf{J}}, \hat{t}_{m n}\right]_{+}\right\}
$$

where $N_{n m}$ is the energy-integrated inter-site Green's function describing the propagation of an electron from site $n$ to $m, \hat{\mathbf{J}}$ is the total moment operator, $t_{m n}$ is the hopping matrix and $[., .]_{+}$ represents an anticommutator (see Supplementary Section IV for details). The method is developed in a many-body form, thus, state-of-the-art numerical approaches such as dynamical meanfield theory can be used to take into account temperature and dynamical Coulomb correlations effects.

The calculated Dzyaloshinskii-Moriya vector equation (2) linking iron atoms 0 and 1 (Fig. 1), for example, is $\mathbf{D}_{01}=(-0.25,0$, $-0.24) \mathrm{meV}$. By symmetry, it lies in the $x z$ plane, perpendicular to the two-fold axis that passes through the oxygen atom ${ }^{8}$. All six symmetrically equivalent vectors have the same $z$ component, but the $x y$ components average to zero. Our calculated canting angle of $0.7^{\circ}$ is only slightly smaller than the experimental value of $0.9^{\circ}$, used for the self-consistent calculation.

Crucially, the sign of the Dzyaloshinskii-Moriya interaction, which we have predicted by two theoretical methods, determines the direction of twist of the magnetic structure, which affects the phase of the magnetic scattering and the sign of the interference term in Fig. 3. It is thus confirmed unambiguously by the experimental data.

In conclusion, we show that a new interference technique in which measurements are carried out with precise control of the amplitude and phase of a reference wave gives an unambiguous result for the sign of the Dzyaloshinskii-Moriya interaction. We find that the twist in the magnetic structure is in the same direction as that of the oxygen atoms. The results prove the efficacy of state-of-the-art electronic structure calculations, able to predict the magnetic ground state and both the direction and strength of the Dzyaloshinskii-Moriya interaction. These findings take us a step closer to realising the prediction of complex non-collinear magnetic structures and the associated properties of an important class of materials that includes weak ferromagnets and multiferroics. Finally we note that, although the current technique would seem to be applicable to a very specific class of materials where the symmetry allows coincidence of magnetic and resonant forbidden scattering, in fact such a coincidence is not particularly rare and includes, among others, all three of the classical weak ferromagnets highlighted by Moriya ${ }^{8}, \alpha-\mathrm{Fe}_{2} \mathrm{O}_{3}, \mathrm{MnCO}_{3}$ and $\mathrm{CoCO}_{3}$, and the high- $T_{\mathrm{C}}$ superconductor parent compound $\mathrm{La}_{2} \mathrm{CuO}_{4}$ (refs 23,26).

\section{Methods}

The experiments were mostly carried out at beamline BM28 (XMaS), European Synchrotron Radiation Facility ${ }^{27}$, with preliminary investigations of the pure magnetic and pure resonant scattering carried out at beamline I16, Diamond Light Source ${ }^{28}$ (see Supplementary Information). Both beamlines provide intense $\mathrm{X}$-ray beams covering the required energy range $(\approx 7 \mathrm{keV})$, focussed onto a $10-800 \mathrm{~K}$ cryofurnace, mounted at the centre of large six-circle diffractometers. The sample-a single crystal of $\mathrm{FeBO}_{3} \sim 4 \times 3 \times 0.05 \mathrm{~mm}^{3}$ in size-was attached with its 001 surface normal to the diffractometer $\phi$ rotation axis ${ }^{27}$, which was parallel to the azimuthal rotation axis, $\psi$ (Fig. 2). Two small rare-earth magnets provided a magnetic field of $0.011 \mathrm{~T}$, sufficient to saturate the weak ferromagnetism within the crystal $x y$ plane, with an orientation determined by the motorized rotation angle of the magnets around the $\phi$ axis. Scattering was in the vertical plane, perpendicular to the linearly $(\sigma)$-polarized incident beam, and a linear polarization analyser, based on a $\mathrm{Cu} 220$ Bragg reflection, selected just the rotated $(\sigma \rightarrow \pi)$ polarization channel. Most measurements were carried out at temperature $T=200 \mathrm{~K}$, where the moments are close to saturation, with subsidiary measurements performed at $T=400 \mathrm{~K}$ (well above the magnetic ordering temperature of $\sim 348 \mathrm{~K}$ ), where the magnetic scattering is absent. The real and imaginary parts of the resonant amplitudes were calculated using FDMNES (Finite Difference Method for Near Edge Structure) package ${ }^{29}$ (see Supplementary Information for details).

Received 31 May 2013; accepted 4 December 2013; published online 9 February 2014 


\section{References}

1. Eerenstein, W., Mathur, N. D. \& Scott, J. F. Multiferroic and magnetoelectric materials. Nature 442, 759-765 (2006).

2. Cheong, S-W. \& Mostovoy, M. Multiferroics: A magnetic twist for ferroelectricity. Nature Mater. 6, 13-20 (2007).

3. Rößler, U. K., Bogdanov, A. N. \& Pfleiderer, C. Spontaneous skyrmion ground states in magnetic metals. Nature 442, 797-801 (2006).

4. Yu, X. Z. et al. Real-space observation of a two-dimensional skyrmion crystal. Nature 465, 901-904 (2010).

5. Dzialoshinskii, I. E. Thermodynamic theory of 'weak' ferromagnetism in antiferromagnetic substances. Sov. Phys. JETP 5, 1259-1262 (1957).

6. Dzyaloshinsky, I. A thermodynamic theory of weak ferromagnetism of antiferromagnetics. J. Phys. Chem. Solids 4, 241-255 (1958).

7. Moriya, T. New mechanism of anisotropic superexchange interaction. Phys. Rev. Lett. 4, 228-230 (1960).

8. Moriya, T. Anisotropic superexchange interaction and weak ferromagnetism. Phys. Rev. 120, 91-98 (1960).

9. Kitzerow, H-S. \& Bahr, C. (eds) Chirality in Liquid Crystals (Springer, 2001).

10. Blundell, S. Magnetism in Condensed Matter (Oxford Univ. Press, 2001).

11. White, R. M. Quantum Theory of Magnetism (Springer, 2007).

12. Matarrese, L. W. \& Stout, J. W. Magnetic anisotropy of $\mathrm{NiF}_{2}$. Phys. Rev. 94, 1792-1793 (1954).

13. Maleyev, S. V. Spin chirality and polarized neutrons. Physica B 350, 26-32 (2004).

14. Okorokov, A. I. et al. The spin chirality in $\mathrm{MnSi}$ single crystal probed by small angle scattering with polarized neutrons. Physica B 350, e323-e326 (2004).

15. Johnson, R. D. et al. X-ray imaging and multiferroic coupling of cycloidal magnetic domains in ferroelectric monodomain $\mathrm{BiFeO}_{3}$. Phys. Rev. Lett. 110, 217206 (2013).

16. Dmitrienko, V. E., Ovchinnikova, E. N., Kokubun, J. \& Ishida, K. Dzyaloshinskii-Moriya interaction: How to measure its sign in weak ferromagnets? JETP Lett. 92, 383-387 (2010).

17. Blume, M. in Resonant Anomalous X-Ray Scattering (eds Materlik, G., Sparks, C. S. \& Fischer, K.) 95-515 (North-Holland, 1994).

18. De Bergevin, F. \& Brunel, M. Diffraction of X-rays by magnetic materials. I. General formulae and measurements on ferro- and ferrimagnetic compounds. Acta Crystallogr. A 37, 314-324 (1981).

19. Dmitrienko, V. E., Ishida, K., Kirfel, A. \& Ovchinnikova, E. N. Polarization anisotropy of X-ray atomic factors and 'forbidden' resonant reflections. Acta Crystallogr. A 61, 481-493 (2005).

20. Lovesey, S. W., Balcar, E., Knight, K. S. \& Rodríguez, J. F. Electronic properties of crystalline materials observed in X-ray diffraction. Phys. Rep. 411, 233-289 (2005).

21. Solovyev, I. V., Lichtenstein, A. I. \& Terakura, K. Is Hunds second rule responsible for the orbital magnetism in solids? Phys. Rev. Lett. 80, 5758-5761 (1998).
22. Shorikov, A. O., Lukoyanov, A. V., Korotin, M. A. \& Anisimov, V. I. Magnetic state and electronic structure of the $\delta$ and $\alpha$ phases of metallic Pu and its compounds. Phys. Rev. B 72, 024458 (2005).

23. Mazurenko, V. V. \& Anisimov, V. I. Weak ferromagnetism in antiferromagnets: $\alpha-\mathrm{Fe}_{2} \mathrm{O}_{3}$ and $\mathrm{La}_{2} \mathrm{CuO}_{4}$. Phys. Rev. B 71, 184434 (2005).

24. Yildirim, T., Harris, A. B., Aharony, A. \& Entin-Wohlman, O. Anisotropic spin Hamiltonians due to spin-orbit and Coulomb exchange interactions. Phys. Rev. B 52, 10239-10267 (1995).

25. Solovyev, I., Hamada, N. \& Terakura, K. Crucial role of the lattice distortion in the magnetism of $\mathrm{LaMnO}_{3}$. Phys. Rev. Lett. 76, 4825-4828 (1996).

26. Katsnelson, M. I., Kvashnin, Y. O., Mazurenko, V. V. \& Lichtenstein, A. I. Correlated band theory of spin and orbital contributions to Dzyaloshinskii-Moriya interactions. Phys. Rev. B 82, 100403 (2010).

27. Brown, S. D. et al. The XMaS beamline at ESRF: Instrumental developments and high resolution diffraction studies. J. Synchrotron Radiat. 8, 1172-1181 (2001)

28. Collins, S. P. et al. Diamond beamline I16 (materials and magnetism). AIP Conf. Proc. 1234, 303-306 (2009).

29. Joly, Y. X-ray absorption near-edge structure calculations beyond the muffin-tin approximation. Phys. Rev. B 63, 125120 (2001).

\section{Acknowledgements}

The work of V.V.M. is supported by the grant program of the President of Russian Federation MK-5565.2013.2, the contracts of the Ministry of Education and Science of Russia No. 14.A18.21.0076 and 14.A18.21.0889. V.E.D. is grateful for the grant of the Presidium RAS No. 24 'Diffraction of synchrotron radiation in multiferroics and chiral magnetics'. M.I.K. acknowledges financial support by FOM (The Netherlands). We thank the staff of ESRF BM-28 for expert assistance, and Y. Shvyd'ko for the loan of the $\mathrm{FeBO}_{3}$ crystal.

\section{Author contributions}

V.E.D. and E.N.O. proposed the project. G.B., S.P.C. and G.N. carried out experimental work at BM-28. S.P.C. carried out experimental work at I16. Y.O.K., V.V.M., A.I.L., M.I.K. performed $a b$ initio calculations. V.E.D. carried out FDMNES calculations.

G.B. performed least-squares fitting of model functions to experimental data. G.N.

carried out multiple scattering simulations. S.P.C., V.E.D. and V.V.M. wrote the majority of the text.

\section{Additional information}

Supplementary information is available in the online version of the paper. Reprints and permissions information is available online at www.nature.com/reprints. Correspondence and requests for materials should be addressed to S.P.C.

\section{Competing financial interests}

The authors declare no competing financial interests. 